\title{
Proteomics paraphernalia
}

\section{Synthesis, analysis and screening.}

Assays-on-Demand

Applied Biosystems

www.europe.appliedbiosystems.com

Ready-to-use genetic screening assays

Applied Biosystems offers 77,000 assays for SNP genotyping and 4,400 assays for quantitative gene expression analysis. All assays are optimized for use on the ABI PRISM 7900Ht, 7700 and 7000 sequence detection systems. The assays are functionally validated and designed specifically for genetic screening.

\section{QIAamp MinElute}

Qiagen

Pure and simple

MinElute kits can be used to isolate viral RNA and DNA simultaneously from a broad range of RNA and DNA viruses. Elution in small volumes yields highly concentrated nucleic acids in less than one hour. Nucleic acids are ready for immediate use in amplification reactions such as PCR and RT-PCR, or for storage at $-20^{\circ} \mathrm{C}$. The kits are suitable for use with plasma, serum and other cellfree body fluids.

\section{ProteinDetector}

KPL www.kpl.com

Alkaline phosphatase ELISA kits

These kits have been designed to offer a convenient starting point for the development of microwell ELISA procedures for antigen or antibody detection. They include pre-tested reagents, including blocking, coating and wash solutions, alkaline phosphataselabelled secondary antibodies, BluePhos substrate and a detailed instruction manual.

\section{Q-Mate}

Qbiogene www.qbiogene.com Use it on and off

The effects of toxic proteins can be studied in vitro and in vivo with this system. Q-Mate uses molecular expression control, allowing researchers to turn expression of the toxic protein off, infect the target cell with the gene of interest, and then turn gene expression back on to study the effects and function of the protein that is expressed. It is based on the regulatory gene $C y m R$, which controls the transformation of $p$-cymene to cumate and the further degradation of the cumate molecule in Pseudomonas putida F1. A recombinant adenovirus is used to carry the gene of interest and infect the target cell.

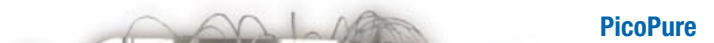

(N Proteomic studies on pure cell populations

PicoPure is a general-purpose chaotrophic buffer validated for protein extraction from laser capture microdissected cells. The kits contain $10 \mathrm{ml}$ of buffer and complete protocols for protein extraction from samples on CapSure LCM caps.

These notes are compiled in the Nature office from information provided by the manufacturers.
PolyPlex

GeneMachines www.genemachines.com Oligonucleotide synthesizer

The newly redesigned PolyPlex 96-well oligonucleotide synthesizer generates a full plate of 20mers in less than three hours. It can synthesize up to one micromole scale using cartridges and accommodating a variety of chemistries, with eight amidite reagent positions. It is compatible with a wide range of reagent bottle sizes.

\section{GenePix 4100A/ Pro 4.1}

Axon Instruments www.axon.com Personal scanner

Axon describes its GenePix 4100A microarray scanner as a compact, easy-to-use device with a high level of performance. It is controlled using GenePix Pro 4.1, a software program for microarray analysis. The program is compatible with all GenePix scanners and can be linked to Acuity, the company's microarray database, data mining and visualization software. It is also available as a stand-alone package for analysis of 16-bit TIFF images from third-party scanners.

\section{GenVision}

DNASTAR

www.dnastar.com

Put words into pictures

This data visualization software creates Postscript images from annotation data stored in text files. Both linear and circular genome maps can be drawn. GenVision is available as a plug-in for Adobe Illustrator for Macs and Windows, or in a stand-alone version for Windows. The plug-in provides dialogs and tools to add GenVision functionality to Illustrator. The stand-alone version provides all of the GenVision image creation functions in a less complex environment. 